\title{
Special issue on current progresses in rubber technology
}

\author{
Manroshan Singh ${ }^{1} \cdot$ Muzafar Zulkifli $^{2}$
}

Published online: 14 January 2022

(C) The Malaysian Rubber Board 2021

Rubber has come a long way since its discovery over 400 years ago. Various rubber-related findings have contributed to both industrial and general applications which serve as an essential part of daily life. Among the most important of these innovations is the vulcanisation process, involving the conversion of a plastic polymer to an elastic material. During the period of WWII when rubber supplies were declining, new synthetic rubbers were invented, and existing rubbers were chemically and physically modified to better fit a variety of purposes. To date, research is still actively being pursued to produce new rubbers and apply them to new functions. In this regard, we are honoured to organize this special issue which emphasizes on materials, methods and technology after the success of the first issue on challenges and methods in experimental investigations and modelling of elastomers organized by Johlitz and Hartmann. We approached researchers working on new aspects of rubber to be considered for publication as part of this special issue (SI).

The SI consists of eleven contributions. The first article focuses on the role of fatty acids and proteins on thermal aging and ozone resistance of natural rubber cured using peroxide vulcanization system (Payungwong, Tuampoemsab, Rojruthai and Sakdapipanich). This is followed by an article by Chen, Zhang, Zhang, Wei, Hu, Luo and Liao which gives some insights on the relationship between coagulation methods on natural rubber and the resulting rubber storage properties. Coagulation produces not only rubber but also odour and serum which is rich in non-rubbers. Pumloifa, Hansupalak, Insuan and Choowongkomon have identified the malodour produced by bacteria in natural rubber and suggested ways of managing the odour in their
Manroshan Singh
manroshan@lgm.gov.my
Muzafar Zulkifli
muzafar@unikl.edu.my
1 Malaysian Rubber Board, Kuala Lumpur, Malaysia
2 Universiti Kuala Lumpur, Kuala Lumpur, Malaysia

article. Similarly, an alternative way of coagulating rubber on a larger scale has been studied by Nik Intan, Siti Salina, Ahmad Kahirul and Yong kok Chong. The new technique integrates the upstream and midstream sectors to increase the smallholders earning by capitalizing on the non-rubber, mainly quebrachitol and proteins, with rubber being the waste material. However, in their current article in this volume, they have shown that the rubber waste from the process is not only cleaner as it contains less dirt compared to SMR 10, but it also has some of the SMR L properties.

On other aspects of rubber, we have two contributors covering biodegradability and sustainability. An article by Aziana, Shabinah Filza, Roslim and Fatimah Rubaizah looks into the biodegradability of epoxidized natural rubber, which is modified from natural rubber and has resistance to chemicals and solvents. For sustainability, we have an article by Zhuo, Zhang, Zhao, Hu, Liao and Liao which looks into rubber from Taraxacum Kok-saghyz. In their work, they have described three ways of extracting the rubber from the roots of the plant.

The remaining five articles are related to some of the many technological developments involving rubber which are currently taking place. The first two are related to the latex form of natural rubber, involving deproteinized latex and cellulose nanofibers. Due to the demand for pillows with improved pressure-relief features, Roslim, Chai, Shamsul, Ho, Fatimah Rubaizah and De Focatiis have successfully developed latex foam pillows from deproteinized natural rubber latex. On cellulose nanofibers, Low, Supramaniam, Abdul Hasif, Tang and Leo showed that the fibers obtained from oil palm empty fruit bunches, can be used as a filler in preparing high-performance rubber composite films for glove manufacturing applications. Articles which address rubber technology in this volume are on thread compounds, energy storage and 3-D printing. As a material having high rubber content, latex reclaim (white reclaim) has been used in the production of premium grade rubber products such as tyres, and retreading materials. In the present article, Nambiathodi, Varghese and Varghese prepared and studied combinations of natural rubber, butadiene rubber and latex 
reclaim to develop cost-efficient tread materials. Rajapaksha, Perera and Vidanapathurana have shown that natural rubber can be used to store energy to replace commercially available toxic polymers. In their article, they fabricated a redox capacitor using a natural rubber (NR)-based solid polymer electrolyte (SPE) and conducting polymer electrodes. The last article in this volume focuses on 3D printing application. In an attempt to develop thermoplastic elastomers as potential 3D printing materials, Dayang Habibah, Nik Intan, Siti Salina, Ngeow, Suhawati and Yong prepared filament materials using ethylene-vinyl acetate and natural rubber for use in fused deposition modelling (FDM).
We would like to thank all authors for these highly interesting contributions. The support given by the Malaysian Rubber Board and Universiti Kuala Lumpur is also greatly appreciated.

Publisher's Note Springer Nature remains neutral with regard to jurisdictional claims in published maps and institutional affiliations. 